\title{
Effects of permanent dipole moments in high-order optical nonlinearity
}

\author{
Luciana C Dávila Romero and David L Andrews† \\ School of Chemical Sciences, University of East Anglia, Norwich NR4 7TJ, UK
}

Received 11 February 1999

\begin{abstract}
High-order optical nonlinearity in molecular systems is of considerable interest, due to the dynamical information it affords, the flexibility in geometry it offers, and due to its involvement in the generation of even harmonics in isotropic media. Here, attention is focused on several processes whose optical response is dominated by two electronic states and which involve the coupling of up to six different optical modes. It is shown that transformation of the electricdipole interaction provides a convenient means for ascertaining the role of intramolecular charge transfer on the high-order nonlinear optics of such systems. The transformation offers calculational expediency and a direct route to expressions for nonlinear molecular polarizabilities. In particular, the method identifies directly terms dependent on various powers of the displacement vector for intramolecular charge transfer.
\end{abstract}

\section{Introduction}

The aim of this work is to expedite analysis of the involvement in high-order optical nonlinearity of the permanent dipole moments in molecular systems, focusing attention on processes involving up to six optical modes. Experimental studies of such high-order interactions [1-9] have recently attracted great interest, mainly for the following reasons: (a) experiments of this type offer dynamical information that is otherwise unavailable [10-16]; (b) they can afford mechanisms for the generation of even harmonics in media that are macroscopically isotropic $[8,17,18]$; (c) they offer considerable scope for the exploitation of resonances; and (d), unlike second harmonic generation (SHG) or sum-frequency generation (SFG), they present a flexibility of geometry. The latter characteristic can be usefully exploited for the fullest characterization of the salient optical nonlinearity.

The signal of any parametric $n$-photon process generated within a molecular medium involves the properties of the radiation and the medium itself. In the electric-dipole approximation, the latter is characterized by the appropriate nonlinear polarizability, which depends on the detailed structure of the molecular electronic states, and also the transition dipole moments connecting pairs of states - at least one of which is virtual. The product of these transition moments connects the initial and final states, which, in a parametric process, are identical (usually the ground state 0 ). The various routes between the initial and the final states necessarily feature diagonal matrix elements, corresponding to permanent dipole moments, as well as off-diagonal terms.

\footnotetext{
$\dagger$ E-mail address: d.1.andrews@uea.ac.uk
} 
The importance in many systems of the permanent dipole moments, though much overlooked in the infancy of the subject, is now well known. The role of such moments is particularly significant in non-centrosymmetric or polar molecules displaying a significant degree of intramolecular charge transfer on electronic excitation - usually quantified in terms of the vector difference between the permanent dipole moments of the excited state and the ground state, traditionally denoted as $\boldsymbol{d}$ [19-21]. Here, we are interested in systems with an optical response dominated by just one electronic excited state $(u)$ and, therefore, only one vector displacement associated with intramolecular charge transfer: such systems are often referred to as two-level systems. In this work the detailed role of permanent moments in several optical processes of high-order nonlinearity is analysed by use of a transformed interaction Hamiltonian, which considerably simplifies the calculations and directly isolates terms dependent on the displacement vector for charge transfer.

\section{Quantum electrodynamical formalism}

The quantum system is formed by the molecules and the electromagnetic field (EM). It is represented by a full quantum electrodynamical (QED) Hamiltonian, which can be described in terms of the Power-Zienau-Woolley formalism [22]. The specific formulation, which is used in the following sections is based on our previous work [23], where the interaction between the EM field and the molecules, $H_{\text {int }}(\xi)$, is considered in the usual electric-dipole approximation.

To analyse an $n$-photon process in a dilute molecular medium we make use of $n$th order perturbation theory. The probability amplitude for the transition from the initial to the final state of the system can be expressed as a sum of terms from each molecule or optically distinct centre:

$$
S=\sum_{\xi=1}^{M} S_{\xi}
$$

where the first non-zero contribution for each molecule is the transition matrix element of the time-evolution operator, $U_{I}$, in the interaction representation

$$
S_{\xi}=\left\langle f\left|U_{I}\right| i\right\rangle_{\xi}=\left\langle f_{\text {sub }}\left|H_{\text {int }}(\xi)\left\{\left(E_{\text {sys }}^{(0)}-H^{(0)}\right)^{-1} H_{\text {int }}(\xi)\right\}^{n-1}\right| i_{\text {sub }}\right\rangle_{\xi} .
$$

By employing the completeness relation identity between the operators

$$
\left\{\left(E_{\text {sys }}^{(0)}-H^{(0)}\right)^{-1} H_{\text {int }}(\xi)\right\}
$$

in equation (2.2), each individual probability amplitude can be expressed as a tensor product of the radiation tensor, $\gamma_{\alpha_{1} \ldots \alpha_{n}}$, and the nonlinear polarizability or high-order hyperpolarizability tensor $O_{\alpha_{1} \ldots \alpha_{n}}^{f i(\eta \omega)}(\xi)$ [24]. The former depends on the polarization vectors of incident photons, and their complex conjugates for emitted photons, multiplied by other beam parameters, which need not concern us here. On the other hand, $O_{\alpha_{1} \ldots \alpha_{n}}^{f i(\eta \omega)}(\xi)$ depends on the differences in energy between the initial state and each of the intermediate states, and on the electric-dipole transition moments, which link the initial and final molecular states through the sequence of virtual intermediate states. In a two-level system there are $2^{n-1}$ such molecular state sequences. It is the nonlinear polarizability $O_{\alpha_{1} \ldots \alpha_{n}}^{f i(\eta \omega)}(\xi)$ that is our present concern. For condensed systems, the optical response can then be cast in terms of the bulk susceptibility $X_{\alpha_{1} \ldots \alpha_{n}}$, through [25]

$$
X_{\alpha_{1} \ldots \alpha_{n}} \equiv \rho L_{\alpha_{1} \ldots \alpha_{2 n}}^{(\eta \omega)} \otimes O_{\alpha_{n+1} \ldots \alpha_{2 n}}^{f i(\eta \omega)}(\xi)
$$


where $\rho$ is the number density and $L_{\alpha_{1} \ldots \alpha_{2 n}}^{(\eta \omega)}$ is a Lorentz factor tensor of rank $2 n$ given by the outer tensor product of $n$ rank-2 tensors;

$$
\boldsymbol{L}^{(\eta \omega)}=\left(\frac{1}{3}\right)^{n} \prod_{i=1}^{n}\left(\kappa_{\omega_{i}}+2 \delta\right) .
$$

Here $\kappa_{\omega_{i}}$ is the dielectric tensor for frequency $\omega_{i}$ and $\delta$ is the Kronecker delta tensor.

For molecular systems, which have an optical response dominated by only two electronic states, it has been shown that a transformed interaction Hamiltonian,

$$
H_{\text {int }}^{\prime}(\xi)=-\left[\underline{\mu}(\xi)-\underline{\mu}^{00}(\xi)\right] \cdot e^{\perp}\left(\boldsymbol{R}_{\xi}\right)
$$

is valid for deriving its optical response [19, 20,26-31]. The subtraction in the square brackets in equation (2.5) is the permanent dipole moment of the initial molecular state, in this case the ground state. The first non-zero contribution to the probability amplitude can properly be expressed in terms of $H_{\text {int }}^{\prime}(\xi)$ as follows:

$$
S_{\xi}=\left\langle f\left|U_{I}\right| i\right\rangle_{\xi}=\left\langle f_{\text {sub }}\left|H_{\text {int }}^{\prime}(\xi)\left\{\left(E_{\text {sys }}^{(0)}-H^{(0)}\right)^{-1} H_{\text {int }}^{\prime}(\xi)\right\}^{n-1}\right| i_{\text {sub }}\right\rangle_{\xi} .
$$

Use of the transformed interaction Hamiltonian has the effect of nullifying the permanent dipole moment of the ground state and recasting the permanent dipole moment of the excited state as the difference between the two [23]. Therefore, as in equation (2.2), the individual probability amplitude can be written as the product of two tensors $\gamma_{\alpha_{1} \ldots \alpha_{n}}$ and $O_{\alpha_{1} \ldots \alpha_{n}}^{\prime f i(\omega)}(\xi)$, where the prime indicates the recast form of the latter tensor in terms of $\left[\mu(\xi)-\underline{\mu}^{00}(\xi)\right]$. Consequently, the number of molecular state sequences linking the initial and final molecular states is substantially reduced from $2^{n-1}$, since all sequences that involve the permanent dipole moment of the initial (ground) state give a null contribution to the nonlinear polarizability.

As will become evident, particular consideration must be given to cases of secular and nonsecular resonance. The latter occur when any molecular excited state differs from the initial state by an amount corresponding to the net energy of a subset of the photons involved. The degree of associated enhancement depends on the magnitude of the damping factors used to extend the theory to dispersive frequency regions. Recent detailed studies [32] based on timereversal symmetry have confirmed that these damping factors should appear with a consistent sign as tilde energies $\tilde{E}_{i_{k} 0}$, representing $\tilde{E}_{i_{k} 0} \equiv\left(E_{i_{k}}-\mathrm{i} \Gamma_{i_{k}}\right)-E_{0}$, where $\Gamma_{i_{k}}$ is the damping factor of the excited state $i_{k}$ (with energy $E_{i_{k}}$ ) and where $E_{0}$ is the energy of the ground state. In secular resonances, a particular subset of photons involved in the process has an overall null energy exchange with the system. In the nonlinear polarizability contribution, for which the intermediate molecular state is identical to the initial state, an apparently divergent response is present; however, the divergent terms are in general present in groups which combine to give a finite (even null) correct response, see, for example, [17].

Before applying the QED formalism to high-order processes, it is helpful to illustrate the procedure by addressing the simple case of three-wave mixing. The analysis of such a process exemplifies the equivalence of equations (2.2) and (2.6) for $n=3$. In the traditional approach, expression (2.2) for any three-photon process has $2^{2}$ possible molecular state sequences; the exponent 2 takes account of the number of intermediate states involved, while the 2 in the base shows that the system is represented by only two electronic states. Specifically, where the two participating states are the ground state 0 and an upper state $u$, the molecular state sequences can be classified by the number $p$ of $0 u(u 0)$ segments involved;

$$
\begin{array}{ll}
p=0: & 0000 \\
p=2: & \text { оии } 0,0 u 00,00 u 0 .
\end{array}
$$

The distinction is significant because the corresponding terms in the nonlinear polarizability entails precisely $p$ transition dipole moments. It is also necessary to consider all the possible 
time orderings that contribute, as represented by distinct Feynman diagrams (FD). In this case there are at the most $3 !=6 \mathrm{FD}$, depending on the number of beams that are involved (for example, in second harmonic generation the degeneracy reduces the number of FD to 3 ). In summary, the tensor characterizing a three-photon parametric process in a two-level system has in general $4 \times 6=24$ different contributions.

To determine the nonlinear polarizability for this process, let us first consider the molecular state sequence that involves only the ground state, 0000 . Here, the hyperpolarizability tensor as given by equation (2.2) has a contribution given by the six time orderings of this particular sequencet;

$$
\sum_{\pi\{1,2,3\}}\left(\frac{\mu_{i_{3}}^{00} \mu_{i_{2}}^{00} \mu_{i_{1}}^{00}}{\left(\eta_{2} \hbar \omega_{2}+\eta_{1} \hbar \omega_{1}\right)\left(\eta_{1} \hbar \omega_{1}\right)}\right)_{\pi}
$$

where $\sum_{\pi\{1,2,3\}}()_{\pi}$ is defined as the sum of all possible permutations of the three modes $\left(\omega_{1}\right.$, $\omega_{2}$ and $\left.\omega_{3}\right)$, and

$$
\eta_{i} \equiv \eta\left(\omega_{i}\right)= \begin{cases}-1 & \text { for photon absorption } \\ +1 & \text { for photon emission. }\end{cases}
$$

After some algebra, expression (2.7) can be written as

$$
\mu_{i_{1}}^{00} \mu_{i_{2}}^{00} \mu_{i_{3}}^{00}\left[\frac{\sum_{i=1}^{3}\left(\eta_{i} \hbar \omega_{i}\right)}{\left(\eta_{3} \hbar \omega_{3}\right)\left(\eta_{2} \hbar \omega_{2}\right)\left(\eta_{1} \hbar \omega_{1}\right)}\right] .
$$

As befits a parametric process, the energy conservation condition implies that the numerator of expression (2.8) is zero, and therefore the susceptibility has no contribution from this sequence.

Of the remaining 18 terms there are six time orderings (FD) for each of the molecular state sequences with $p=2$. Using the sum notation introduced in expression (2.7) we can write all these terms in a compact form:

$$
\begin{gathered}
\sum_{\pi\{1,2,3\}}\left(\frac{\mu_{i_{3}}^{0 u} \mu_{i_{2}}^{u u} \mu_{i_{1}}^{u 0}}{\left(E+\eta_{2} \hbar \omega_{2}+\eta_{1} \hbar \omega_{1}\right)\left(E+\eta_{1} \hbar \omega_{1}\right)}+\frac{\mu_{\pi\{3\}}^{0 u} \mu_{\pi\{1\}}^{u 0} \mu_{\pi\{2\}}^{00}}{\left(E+\eta_{1} \hbar \omega_{1}+\eta_{2} \hbar \omega_{2}\right)\left(\eta_{2} \hbar \omega_{2}\right)}\right. \\
\left.+\frac{\mu_{i_{2}}^{00} \mu_{i_{3}}^{0 u} \mu_{i_{1}}^{u 0}}{\left(\eta_{3} \hbar \omega_{3}+\eta_{1} \hbar \omega_{1}\right)\left(E+\eta_{1} \hbar \omega_{1}\right)}\right)_{\pi}
\end{gathered}
$$

where $E \equiv E_{u 0}=E_{u}-E_{0}$. Equation (2.9) can be re-expressed as

$$
\begin{gathered}
\sum_{\pi\{1,2,3\}}\left(\frac{\mu_{i_{3}}^{0 u} d_{i_{2}} \mu_{i_{1}}^{u 0}}{\left(E+\eta_{2} \hbar \omega_{2}+\eta_{1} \hbar \omega_{1}\right)\left(E+\eta_{1} \hbar \omega_{1}\right)}+\frac{\mu_{i i_{3}}^{0 u} \mu_{i_{1}}^{u 0} \mu_{i_{2}}^{00}}{\left(E+\eta_{1} \hbar \omega_{1}\right)\left(\eta_{2} \hbar \omega_{2}\right)}\right. \\
\left.+\frac{\mu_{i_{2}}^{00} \mu_{i_{3}}^{0 u} \mu_{i_{1}}^{u 0}}{\left(\eta_{3} \hbar \omega_{3}+\eta_{1} \hbar \omega_{1}\right)\left(E+\eta_{1} \hbar \omega_{1}\right)}\right)_{\pi} \\
=\sum_{\pi\{1,2,3\}}\left(\frac{\mu_{i_{3}}^{0 u} d_{i_{2}} \mu_{i_{1}}^{u 0}}{\left(E+\eta_{2} \hbar \omega_{2}+\eta_{1} \hbar \omega_{1}\right)\left(E+\eta_{1} \hbar \omega_{1}\right)}\right. \\
\left.+\frac{\mu_{i_{3}}^{0 u} \mu_{i_{1}}^{u 0} \mu_{i_{2}}^{00} \sum_{i=1}^{3}\left(\eta_{i} \hbar \omega_{i}\right)}{\left(\eta_{3} \hbar \omega_{3}+\eta_{1} \hbar \omega_{1}\right)\left(E+\eta_{1} \hbar \omega_{1}\right)\left(\eta_{2} \hbar \omega_{2}\right)}\right)_{\pi}
\end{gathered}
$$

and the last term in equation (2.10) is again zero because of energy conservation. As a result, equations (2.8) and (2.10) together show that for the three-photon process the nonlinear polarizability tensor can be simply expressed as

$$
\sum_{\pi\{1,2,3\}}\left(\frac{\mu_{i_{3}}^{0 u} d_{i_{2}} \mu_{i_{1}}^{u 0}}{\left(E+\eta_{2} \hbar \omega_{2}+\eta_{1} \hbar \omega_{1}\right)\left(E+\eta_{1} \hbar \omega_{1}\right)}\right)_{\pi}
$$

$\dagger$ This expression is proportional to the coefficient $L_{3,3}^{00}$ of [23]. 
which is consistent with the form obtained directly by use of the transformed interaction Hamiltonian in (2.6), as shown in [23]. It can be seen that the only state sequence that contributes to the final form of the hyperpolarizability tensor, expression (2.11), is the one which does not involve a 00 segment $(0 u u 0)$, and in which the $u u$ segment is related to the $\boldsymbol{d}=\mu^{u u}-\mu^{00}$ vector. This rule can be followed in any $n$-photon process.

For cases involving higher numbers of photons the calculation of the hyperpolarizability tensor, through equation (2.2), becomes increasingly difficult. The main complication when dealing with systems involving $n \geqslant 3$ photons is that the number of sequences and FDs increases rapidly, giving a total of $2^{(n-1)} n$ !, all of which give net contribution to $O_{\alpha_{1} \ldots \alpha_{n}}^{f i(\eta \omega)}(\xi)$. On the other hand, when the transformed interaction Hamiltonian (2.5) is used, the number of sequences, which give a net contribution is reduced drastically, allowing the derivation of expressions for the salient nonlinear polarizability tensors in a direct and simple manner. In particular, this method facilitates the identification of terms involving a linear or higher-order dependence on $\boldsymbol{d}$, the vector signifying the extent of intramolecular charge transfer on optical excitation.

\section{High-order processes in two-level systems}

The equivalence between the result of implementing the two interaction Hamiltonians expedites direct and concise formulation of the nonlinear polarizability tensors for four-, five- and sixwave mixing processes. Hence instead of using all $2^{n-1}$ molecular sequences to determine the detailed tensor structure, the following simple algorithm can be used to obtain the expressions given by the transformed interaction Hamiltonian [23]:

$$
\underline{\mu}^{u u} \mapsto \underline{\mu}^{u u}-\underline{\mu}^{00}=d \quad \underline{\mu}^{00} \mapsto 0
$$

whilst the transition dipole moments remain unaltered. This algorithm therefore allows us to discard any contribution associated with a molecular state sequence that involves the segment 00 , as long as the segment $u u$ is appropriately reinterpreted.

\subsection{Four-wave mixing}

In this process there are $2^{3}=8$ molecular state sequences and $4 !=24$ time orderings for each sequence. As in the three-wave mixing process, these molecular state sequences can be classified by the number of $0 u$ segments involved $(p)$;

$$
\begin{array}{ll}
p=0: & 00000 \\
p=2: & 0 и и и 0,0 и и 00,00 и и 0,0 u 000,00 u 00,000 u 0 \\
p=4: & \text { ои0u } 0 .
\end{array}
$$

By application of the algorithm, it transpires that only sequences $0 u u u 0$ and $0 u 0 u 0$, which have no permanent dipole moment $\mu^{00}$ involved, contribute to the nonlinear polarizability tensor, which thereby assumes the following form:

$$
\begin{gathered}
\sum_{\pi\{1,2,3,4\}}\left(\frac{\mu_{i_{4}}^{0 u} d_{i_{3}} d_{i_{2}} \mu_{i_{1}}^{u 0}}{\left(E+\eta_{3} \hbar \omega_{3}+\eta_{2} \hbar \omega_{2}+\eta_{1} \hbar \omega_{1}\right)\left(E+\eta_{2} \hbar \omega_{2}+\eta_{1} \hbar \omega_{1}\right)\left(E+\eta_{1} \hbar \omega_{1}\right)}\right. \\
\left.+\frac{\mu_{i_{4}}^{0 u} \mu_{i_{3}}^{u 0} \mu_{i_{2}}^{0 u} \mu_{i_{1}}^{u 0}}{\left(E+\eta_{3} \hbar \omega_{3}+\eta_{2} \hbar \omega_{2}+\eta_{1} \hbar \omega_{1}\right)\left(\eta_{2} \hbar \omega_{2}+\eta_{1} \hbar \omega_{1}\right)\left(E+\eta_{1} \hbar \omega_{1}\right)}\right)_{\pi}
\end{gathered}
$$

In the compact form given by (3.2) it is obvious that in the particular case where $|d| \gg\left|\mu^{u 0}\right|$ the first term becomes dominant and the second can be neglected, and vice versa for $|d| \ll\left|\underline{\mu}^{u 0}\right|$. 
In the latter case, special consideration must be taken since secular resonance can occur if two of the four photons involved in the process satisfy the relation $\left(\eta_{i} \hbar \omega_{i}+\eta_{j} \hbar \omega_{j}\right)=0$, for $\forall i, j$ such that $i, j \in\{1,2,3,4\}$. In that case, the susceptibility tensor as written in (3.2) presents singularities in the second term, which must be addressed carefully. It can also be seen from equation (3.2) that, for $\boldsymbol{d}=\mathbf{0}$ (i.e. $\mu^{u u}=\mu^{00}$ ), the hyperpolarizability tensor is a null tensor only if the transition dipole moment is also zero. This characteristic is a feature of any even-photon process [23].

3.1.1. Particular case. A particular case of four-wave mixing is a process involving two laser beams, where the system absorbs from one beam two photons of frequency $\omega$, while the second beam of frequency $\omega^{\prime}$ acts as a pump producing stimulated emission. As a result, a signal is produced with frequency $\omega^{\prime \prime}$ which, since the process is parametric, satisfies the condition $\omega^{\prime \prime}=2 \omega-\omega^{\prime}$. In passing, we note that this process can offer a distinctive rate enhancement when the frequency difference $\left|\omega-\omega^{\prime}\right|$ coincides with a Raman-active frequency of the medium. However, to appropriately describe this widely studied case, known as coherent anti-Stokes Raman scattering (CARS), it is better to cast the theory in terms of a three-level system, for which the expression given in (3.2) is no longer representative. We shall therefore limit our study to the case where $\left|\omega-\omega^{\prime}\right|$ is not a Raman-active frequency. Table 1 summarizes the characteristics of the photons involved. Although in this table the propagation vectors are included to fully specify the process, it is important to notice that for the calculation of the nonlinear polarizability tensor, here or for any $n$-photon process related to dipolar coupling, this data are superfluous. For the particular case under consideration it is readily seen that the condition $\left(\eta_{i} \hbar \omega_{i}+\eta_{j} \hbar \omega_{j}\right)=0$ is not satisfied for any pair $i, j \in\{1,2,3,4\}$, and consequently there is no secular resonance.

Table 1. Photon characteristics for a particular four-wave mixing process (the angle between $\hat{k}$ and $\hat{\boldsymbol{k}}^{\prime}$ is arbitrary, and the labels $\lambda, \lambda^{\prime}$ and $\lambda^{\prime \prime}$ refer to the polarizations of the beams). The frequencies of the beams involved satisfy the energy conservation condition $\omega^{\prime \prime}=2 \omega-\omega^{\prime}$.

\begin{tabular}{llll}
\hline & Frequency & & Direction of propagation \\
\hline Absorbed photons & & & \\
$(\boldsymbol{k}, \lambda)$ & $\omega_{1}=\omega$ & $\eta_{1}=1$ & $\hat{\boldsymbol{k}}$ \\
$(\boldsymbol{k}, \lambda)$ & $\omega_{2}=\omega$ & $\eta_{2}=1$ & $-\hat{\boldsymbol{k}}$ \\
Emitted photons & & & \\
$\left(\boldsymbol{k}^{\prime}, \lambda^{\prime}\right)$ & $\omega_{3}=\omega^{\prime}$ & $\eta_{3}=-1$ & $\hat{\boldsymbol{k}}^{\prime}$ \\
$\left(\boldsymbol{k}^{\prime \prime}, \lambda^{\prime \prime}\right)$ & $\omega_{4}=\omega^{\prime \prime}$ & $\eta_{4}=-1$ & $-\hat{\boldsymbol{k}}^{\prime}$ \\
\hline
\end{tabular}

An expression for the nonlinear susceptibility tensor can now be written as a sum of five different terms with numerators: $\mu_{i}^{0 u} d_{j} d_{k} \mu_{l}^{u 0}, \mu_{j}^{0 u} d_{i} d_{l} \mu_{k}^{u 0}, \mu_{i}^{0 u} d_{k} d_{l} \mu_{j}^{u 0}, \mu_{k}^{0 u} d_{i} d_{j} \mu_{l}^{u 0}$ and $\mu_{i}^{0 u} \mu_{j}^{u 0} \mu_{k}^{0 u} \mu_{l}^{u 0}$. The result of combining the different contributions is

$$
\begin{aligned}
O_{i j k l}^{(3)}\left(\omega^{\prime \prime} ;-\omega^{\prime}\right. & , \omega, \omega) \\
\equiv & O_{i j(k l)}^{(3)}\left(\omega^{\prime \prime} ;-\omega^{\prime}, \omega, \omega\right) \\
= & \left(\mu_{i}^{0 u} d_{j} d_{k} \mu_{l}^{u 0}+\mu_{i}^{0 u} d_{j} d_{l} \mu_{k}^{u 0}\right)\left[\left\{2 E \left(2 E^{4}+E_{u 0}^{2}\left(8 \hbar^{2} \omega^{2}-6 \hbar^{2} \omega \omega^{\prime}\right)\right.\right.\right. \\
& \left.\left.+39 \hbar^{4} \omega^{3} \omega^{\prime}+3 \hbar^{4} \omega \omega^{\prime 3}-18 \hbar^{4} \omega^{2} \omega^{\prime 2}-28 \hbar^{4} \omega^{4}\right)\right\}\left\{\left(E^{2}-\left(2 \hbar \omega-\hbar \omega^{\prime}\right)^{2}\right)\right. \\
& \left.\left.\times\left(E^{2}-\left(\hbar \omega-\hbar \omega^{\prime}\right)^{2}\right)\left(E^{2}-4(\hbar \omega)^{2}\right)\left(E^{2}-(\hbar \omega)^{2}\right)\right\}^{-1}\right] \\
& +\left(\mu_{j}^{0 u} d_{i} d_{k} \mu_{l}^{u 0}+\mu_{j}^{0 u} d_{i} d_{l} \mu_{k}^{u 0}\right)\left[\left\{2 E \left(2 E^{4}+E^{2}\left(-4 \hbar^{2} \omega^{2}+6 \hbar^{2} \omega \omega^{\prime}\right)\right.\right.\right.
\end{aligned}
$$




$$
\begin{aligned}
& \left.\left.-3 \hbar^{4} \omega^{3} \omega^{\prime}-3 \hbar^{4} \omega \omega^{\prime 3}+2 \hbar^{4} \omega^{4}\right)\right\}\left\{\left(E^{2}-\left(\hbar \omega-\hbar \omega^{\prime}\right)^{2}\right)\left(E^{2}-(\hbar \omega)^{2}\right)\right. \\
& \left.\left.\times\left(E^{2}-\left(\hbar \omega^{\prime}\right)^{2}\right)\left(E^{2}-4(\hbar \omega)^{2}\right)\right\}^{-1}\right] \\
& +\mu_{i}^{0 u} d_{k} d_{l} \mu_{j}^{u 0}\left[\frac{4 E\left(E^{2}+3 \hbar^{2} \omega^{\prime 2}-6 \hbar^{2} \omega \omega^{\prime}-2 \hbar^{2} \omega^{2}\right)}{\left(E^{2}-\left(2 \hbar \omega-\hbar \omega^{\prime}\right)^{2}\right)\left(E^{2}-\left(\hbar \omega-\hbar \omega^{\prime}\right)^{2}\right)\left(E^{2}-\left(\hbar \omega^{\prime}\right)^{2}\right)}\right] \\
& +\mu_{k}^{0 u} d_{i} d_{j} \mu_{l}^{u 0}\left[\frac{4 E}{\left(E^{2}-\left(\hbar \omega-\hbar \omega^{\prime}\right)^{2}\right)\left(E^{2}-(\hbar \omega)^{2}\right)}\right] \\
& +\mu_{i}^{0 u} \mu_{j}^{u 0} \mu_{k}^{0 u} \mu_{l}^{u 0}\left[\frac{4 E\left(E^{2}\left(3 \hbar \omega+\hbar \omega^{\prime}\right)-\hbar^{3} \omega^{\prime 3}+\hbar^{3} \omega \omega^{\prime 2}+4 \hbar^{4} \omega^{2} \omega^{\prime}\right)}{(\hbar \omega)\left(E^{2}-\left(2 \hbar \omega-\hbar \omega^{\prime}\right)^{2}\right)\left(E^{2}-(\hbar \omega)^{2}\right)\left(E^{2}-\left(\hbar \omega^{\prime}\right)^{2}\right)}\right]
\end{aligned}
$$

The symmetry of the tensor under the interchange of the sub-indices $(k l)$ is due to the fact that there are two photons in the process with identical frequency.

It is interesting to consider the case of electronic resonance, accommodated by inclusion of damping; $E \equiv E_{u}-E_{0} \rightarrow \tilde{E}=\left(E_{u}-E_{0}\right)-\mathrm{i} \Gamma$, where $\Gamma / 2 \hbar$ is the frequency linewidth of the state $u$. Then, when any of the following conditions is satisfied: $E \approx \hbar \omega, E \approx \hbar \omega^{\prime}$, $E \approx 2 \hbar \omega, E \approx 2 \hbar \omega-\hbar \omega^{\prime}$ or $E \approx \hbar \omega-\hbar \omega^{\prime}$; the system will exhibit resonance behaviour. In each case, certain terms become dominant and the expression of $O_{i j(k l)}^{(3)}\left(\omega^{\prime \prime} ;-\omega^{\prime}, \omega, \omega\right)$ is greatly simplified. In table 2 the different cases are tabulated, in each case the dominant terms being inversely proportional to the damping factor $\Gamma$.

Table 2. Dominant terms in resonance four-wave mixing.

\begin{tabular}{lll}
\hline Resonance at & $\Gamma^{-l}$ & \\
\hline$E \sim 2 \hbar \omega-\hbar \omega^{\prime}$ & $l=1$ & $\begin{array}{l}\mu_{i}^{0 u} d_{j} d_{k} \mu_{l}^{u 0} ; \mu_{i}^{0 u} d_{j} d_{l} \mu_{k}^{u 0} ; \mu_{i}^{0 u} d_{k} d_{l} \mu_{j}^{u 0} ; \\
\mu_{i}^{0 u} \mu_{j}^{u 0} \mu_{k}^{0 u} \mu_{l}^{u 0}\end{array}$ \\
$E \sim \hbar \omega-\hbar \omega^{\prime}$ & $l=1$ & $\begin{array}{l}\mu_{i}^{0 u} d_{j} d_{k} \mu_{l}^{u 0} ; \mu_{i}^{0 u} d_{j} d_{l} \mu_{k}^{u 0} ; \mu_{j}^{0 u} d_{i} d_{k} \mu_{l}^{u 0} ; \\
\mu_{j}^{0 u} d_{i} d_{l} \mu_{k}^{u 0} ; \mu_{i}^{0 u} d_{k} d_{l} \mu_{j}^{u 0} ; \mu_{k}^{0 u} d_{i} d_{j} \mu_{l}^{u 0}\end{array}$ \\
$E \sim 2 \hbar \omega$ & $l=1$ & $\begin{array}{l}\mu_{i}^{0 u} d_{j} d_{k} \mu_{l}^{u 0} ; \mu_{i}^{0 u} d_{j} d_{l} \mu_{k}^{u 0} ; \mu_{j}^{0 u} d_{i} d_{k} \mu_{l}^{u 0} ; \\
\mu_{j}^{0 u} d_{i} d_{l} \mu_{k}^{u 0}\end{array}$ \\
$E \sim \hbar \omega$ & $l=1$ & $\begin{array}{l}\mu_{i}^{0 u} d_{j} d_{k} \mu_{l}^{u 0} ; \mu_{i}^{0 u} d_{j} d_{l} \mu_{k}^{u 0} ; \mu_{j}^{0 u} d_{i} d_{k} \mu_{l}^{u 0} ; \\
\mu_{j}^{0 u} d_{i} d_{l} \mu_{k}^{u 0} ; \mu_{k}^{0 u} d_{i} d_{j} \mu_{l}^{u 0} ; \mu_{i}^{0 u} \mu_{j}^{u 0} \mu_{k}^{0 u} \mu_{l}^{u 0}\end{array}$ \\
$E \sim \hbar \omega^{\prime}$ & $\begin{array}{l}\mu_{j}^{0 u} d_{i} d_{k} \mu_{l}^{u 0} ; \mu_{j}^{0 u} d_{i} d_{l} \mu_{k}^{u 0} ; \mu_{i}^{0 u} d_{k} d_{l} \mu_{j}^{u 0} ; \\
\mu_{i}^{0 u} \mu_{j}^{u 0} \mu_{k}^{0 u} \mu_{l}^{u 0}\end{array}$ \\
\hline
\end{tabular}

\subsection{Five-wave mixing}

For this case there are $2^{4}=16$ molecular state sequences and 120 (5!) FDs, which are again classified in terms of the number of transition dipole moments

$p=0: \quad 000000$

$p=2:$ Оииии0, Оиии00, О0иии0, Оии000, 00ии00, 000ии0, 0u0000, 00u000, 000u00,

$000 u 0$

$p=4: \quad 0 u и 0 u 0,0 u 0 u и 0,0 u 0 u 00,00 u 0 u 0,0 u 00 u 0$.

It can be readily seen, by applying the algorithm given in (3.1), that the sequences, which give a finite contribution to the nonlinear polarizability tensor are $0 u и u и 0,0 u u 0 u 0$ and $0 u 0 u u 0$. 
The same result is obtained, after some quite intricate algebra, when the traditional interaction Hamiltonian is used. By either method the following result is obtained:

$$
\begin{aligned}
\sum_{\pi\{1,2,3,4,5\}}\left(\frac{\mu_{i_{5}}^{0 u} d_{i_{4}} d_{i_{3}} d_{i_{2}} \mu_{i_{1}}^{u 0}}{\left(E+\sum_{i=1}^{4} \eta_{i} \hbar \omega_{i}\right)\left(E+\sum_{i=1}^{3} \eta_{i} \hbar \omega_{i}\right)\left(E+\sum_{i=1}^{2} \eta_{i} \hbar \omega_{i}\right)\left(E+\eta_{1} \hbar \omega_{1}\right)}\right. \\
+\frac{\mu_{i_{5}}^{0 u} d_{i_{4}} \mu_{i_{3}}^{u 0} \mu_{i_{2}}^{0 u} \mu_{i_{1}}^{u 0}}{\left(E+\sum_{i=1}^{4} \eta_{i} \hbar \omega_{i}\right)\left(E+\sum_{i=1}^{3} \eta_{i} \hbar \omega_{i}\right)\left(\sum_{i=1}^{2} \eta_{i} \hbar \omega_{i}\right)\left(E+\eta_{1} \hbar \omega_{1}\right)} \\
\left.+\frac{\mu_{i_{5}}^{0 u} \mu_{i_{4}}^{u 0} \mu_{i_{3}}^{0 u} d_{i_{2}} \mu_{i_{1}}^{u 0}}{\left(E+\sum_{i=1}^{4} \eta_{i} \hbar \omega_{i}\right)\left(\sum_{i=1}^{3} \eta_{i} \hbar \omega_{i}\right)\left(E+\sum_{i=1}^{2} \eta_{i} \hbar \omega_{i}\right)\left(E+\eta_{1} \hbar \omega_{1}\right)}\right)_{\pi}
\end{aligned}
$$

Since the process involves an odd number of photons, the nonlinear response is forbidden when $\boldsymbol{d}=0$. In the particular case where $|\boldsymbol{d}| \gg\left|\underline{\mu}^{u 0}\right|$, the main contribution to the tensor is given by the first term. From equation (3.4) it can also be seen that singularities may be presented by the second and third terms, for particular combinations of the frequencies involved. This will occur whenever the photons involved satisfy

$$
\left(\sum_{i=1}^{2} \eta_{i} \hbar \omega_{i}\right)_{\pi} \cong 0 \quad \text { and/or } \quad\left(\sum_{i=1}^{3} \eta_{i} \hbar \omega_{i}\right)_{\pi} \cong 0 .
$$

An example where these divergences are present is studied in the following subsection.

3.2.1. Particular case. Here we focus on a particular five-wave mixing process studied in previous works [5-7], in those cases without consideration of secularity or the features associated with two-level response. This system involves two different lasers, one of which is used to generate two counterpropagating modes. A summary of the characteristics of each beam is given in table 3 and a more extensive description of the process can be found in [5-7].

Table 3. Photon characteristics for a particular five-wave mixing process (the angle between $\hat{k}$ and $\hat{k}^{\prime}$ is arbitrary, and the labels $\lambda, \lambda^{\prime}, \tilde{\lambda}$ and $\lambda^{\prime \prime}$ refer to the polarizations of the beams). The frequencies of the beams involved satisfy the energy conservation condition $\omega^{\prime \prime}=\omega+\omega^{\prime}$.

\begin{tabular}{llll}
\hline & Frequency & & Direction of propagation \\
\hline Absorbed photons & & & \\
$(\boldsymbol{k}, \lambda)$ & $\omega_{1}=\omega$ & $\eta_{1}=1$ & $\hat{\boldsymbol{k}}$ \\
$(-\boldsymbol{k}, \lambda)$ & $\omega_{2}=\omega$ & $\eta_{2}=1$ & $-\hat{\boldsymbol{k}}$ \\
$\left(\boldsymbol{k}^{\prime}, \lambda^{\prime}\right)$ & $\omega_{3}=\omega^{\prime}$ & $\eta_{3}=1$ & $\hat{\boldsymbol{k}}^{\prime}$ \\
Emitted photons & & & \\
$(\tilde{\boldsymbol{k}}, \tilde{\lambda})$ & $\omega_{4}=\omega$ & $\eta_{4}=-1$ & $-\hat{\boldsymbol{k}}^{\prime}$ \\
$\left(\boldsymbol{k}^{\prime \prime}, \lambda^{\prime \prime}\right)$ & $\omega_{5}=\omega^{\prime \prime}$ & $\eta_{5}=-1$ & $\hat{\boldsymbol{k}}^{\prime}$ \\
\hline
\end{tabular}

For this particular five-wave process, the second term of the polarizability tensor will present secular resonances when

$$
\left(\sum_{i=1}^{2} \eta_{i} \hbar \omega_{i}\right)_{\pi}=\eta_{1,2} \hbar \omega_{1,2}+\eta_{4} \hbar \omega_{4}
$$

while for the third term this problem will arise when

$$
\left(\sum_{i=1}^{3} \eta_{i} \hbar \omega_{i}\right)_{\pi}=\eta_{1,2} \hbar \omega_{1,2}+\eta_{3} \hbar \omega_{3}+\eta_{5} \hbar \omega_{5} .
$$


There are 12 terms for each type of singularity. Hence, the nonlinear polarizability can be written as a sum of two terms:

$$
O^{(4)}=O_{\text {non-sec }}^{(4)}+O_{\mathrm{sec}}^{(4)} \text {. }
$$

The first term of equation (3.4) contributes only to the non-secular part, $O_{\text {non-sec }}^{(4)}$ in (3.7), while the second and third terms of (3.4) contribute to both secular and non-secular terms in (3.7). Then, $O_{\text {non-sec }}^{(4)}$ is

$$
\begin{aligned}
& \sum_{\pi\{1,2,3,4,5\}}\left(\frac{\mu_{i_{5}}^{0 u} d_{i_{4}} d_{i_{3}} d_{i_{2}} \mu_{1_{1}}^{u 0}}{\left(E+\sum_{i=1}^{4} \eta_{i} \hbar \omega_{i}\right)\left(E+\sum_{i=1}^{3} \eta_{i} \hbar \omega_{i}\right)\left(E+\sum_{i=1}^{2} \eta_{i} \hbar \omega_{i}\right)\left(E+\eta_{1} \hbar \omega_{1}\right)}\right)_{\pi} \\
& +\sum_{\substack{\text { non-sec } \\
\pi\{1,2,3,4,5\}}}\left(\frac{\mu_{i_{5}}^{0 u} d_{i_{4}} \mu_{i_{3}}^{u 0} \mu_{i_{2}}^{0 u} \mu_{i_{1}}^{u 0}}{\left(E+\sum_{i=1}^{4} \eta_{i} \hbar \omega_{i}\right)\left(E+\sum_{i=1}^{3} \eta_{i} \hbar \omega_{i}\right)\left(\sum_{i=1}^{2} \eta_{i} \hbar \omega_{i}\right)\left(E+\eta_{1} \hbar \omega_{1}\right)}\right. \\
& \left.+\frac{\mu_{i_{5}}^{0 u} \mu_{i_{4}}^{u 0} \mu_{i_{3}}^{0 u} d_{i_{2}} \mu_{i_{1}}^{u 0}}{\left(E+\sum_{i=1}^{4} \eta_{i} \hbar \omega_{i}\right)\left(\sum_{i=1}^{3} \eta_{i} \hbar \omega_{i}\right)\left(E+\sum_{i=1}^{2} \eta_{i} \hbar \omega_{i}\right)\left(E+\eta_{1} \hbar \omega_{1}\right)}\right)_{\pi} .
\end{aligned}
$$

To address the singularities present in $O_{\mathrm{sec}}^{(4)}$, it is expedient to introduce a slight variation of the frequencies of the anti-parallel beams [17]:

$$
\begin{array}{ll}
\omega_{1}=\omega \rightarrow \omega+\delta & \omega_{2}=\omega \rightarrow \omega-\delta \\
\omega_{3}=\omega^{\prime} \rightarrow \omega^{\prime} & \omega_{4}=\omega \rightarrow \omega
\end{array}
$$

When the limit of $\delta \rightarrow 0$ is taken, a finite combination of the terms leading to singularities is effected, and a well ordered result for the five-wave mixing process of interest is recovered. All 48 terms are tackled in a similar manner. For instance, let us consider the FD that corresponds to the time ordering $\pi\{1,2,3,4,5\} \rightarrow\left(\omega_{2}, \omega_{3}, \omega_{5}, \omega_{1}, \omega_{4}\right)$ and sum with its counterpart, where $\omega_{1}$ and $\omega_{2}$ are interchanged,

$$
\begin{aligned}
(E-\hbar \omega)^{-1}( & -\hbar \delta)^{-1}\left(E-\hbar \omega-\hbar \delta-\hbar \omega^{\prime}\right)^{-1}(E-\hbar \omega-\hbar \delta)^{-1} \mu_{i_{4}}^{0 u} \mu_{i_{2}}^{u 0} \mu_{i_{5}}^{0 u} d_{i_{3}} \mu_{i_{1}}^{u 0} \\
& +(E-\hbar \omega)^{-1}(\hbar \delta)^{-1}\left(E-\hbar \omega+\hbar \delta-\hbar \omega^{\prime}\right)^{-1}(E-\hbar \omega+\hbar \delta)^{-1} \\
& \times \mu_{i_{4}}^{0 u} \mu_{i_{1}}^{u 0} \mu_{i_{5}}^{0 u} d_{i_{3}} \mu_{i_{2}}^{u 0} .
\end{aligned}
$$

Factorizing and taking the limit of $\delta \rightarrow 0$, we can rewrite the expression as

$\mu_{i_{4}}^{0 u} \mu_{i_{1}}^{u 0} \mu_{i_{5}}^{0 u} d_{i_{3}} \mu_{i_{2}}^{u 0}\left(E-\hbar \omega-\hbar \omega^{\prime}\right)^{-1}(E-\hbar \omega)^{-2}(-2)\left\{\left(E-\hbar \omega-\hbar \omega^{\prime}\right)^{-1}+(E-\hbar \omega)^{-1}\right\}$

and the apparent singularity is removed. Proceeding in this way, the final and complete result for the secular part of the nonlinear susceptibility is

$$
\begin{aligned}
O_{\mathrm{sec}}^{(4)}=(-1)( & \left.\mu_{i_{5}}^{0 u} d_{i_{2}} \mu_{i_{3}}^{u 0} \mu_{i_{4}}^{0 u} \mu_{i_{1}}^{u 0}+\mu_{i_{5}}^{0 u} d_{i_{1}} \mu_{i_{3}}^{u 0} \mu_{i_{4}}^{0 u} \mu_{i_{2}}^{u 0}\right) \\
& \times\left\{\left(E-\hbar \omega-\hbar \omega^{\prime}\right)^{-1}\left(E-\hbar \omega^{\prime}\right)^{-1}(E-\hbar \omega)^{-1}\right. \\
& \times\left[\left(E-\hbar \omega-\hbar \omega^{\prime}\right)^{-1}+\left(E-\hbar \omega^{\prime}\right)^{-1}+(E-\hbar \omega)^{-1}\right] \\
& +\left(E-\hbar \omega-\hbar \omega^{\prime}\right)^{-1}\left(E-\hbar \omega^{\prime}\right)^{-1}(E+\hbar \omega)^{-1} \\
& \times\left[\left(E-\hbar \omega-\hbar \omega^{\prime}\right)^{-1}+\left(E-\hbar \omega^{\prime}\right)^{-1}+(E+\hbar \omega)^{-1}\right] \\
& +\left(E+\hbar \omega+\hbar \omega^{\prime}\right)^{-1}\left(E+\hbar \omega^{\prime}\right)^{-1}(E-\hbar \omega)^{-1} \\
& \times\left[\left(E+\hbar \omega+\hbar \omega^{\prime}\right)^{-1}+\left(E+\hbar \omega^{\prime}\right)^{-1}+(E-\hbar \omega)^{-1}\right] \\
& +\left(E+\hbar \omega+\hbar \omega^{\prime}\right)^{-1}\left(E+\hbar \omega^{\prime}\right)^{-1}(E+\hbar \omega)^{-1} \\
& \left.\times\left[\left(E+\hbar \omega+\hbar \omega^{\prime}\right)^{-1}+\left(E+\hbar \omega^{\prime}\right)^{-1}+(E+\hbar \omega)^{-1}\right]\right\}
\end{aligned}
$$




$$
\begin{aligned}
& +(-2)\left(\mu_{i_{3}}^{0 u} d_{i_{5}} \mu_{i_{2}}^{u 0} \mu_{i_{4}}^{0 u} \mu_{i_{1}}^{u 0}\right) \\
& \times\left\{\left(E+\hbar \omega^{\prime}\right)^{-1}(E-\hbar)^{-2}\left[\left(E+\hbar \omega^{\prime}\right)^{-1}+2\left(E-\hbar \omega^{\prime}\right)^{-1}\right]\right. \\
& +\left(E+\hbar \omega^{\prime}\right)^{-1}(E-\hbar \omega)^{-1}(E+\hbar \omega)^{-1} \\
& \times\left[\left(E+\hbar \omega^{\prime}\right)^{-1}+(E-\hbar \omega)^{-1}+(E+\hbar \omega)^{-1}\right] \\
& +\left(E-\hbar \omega^{\prime}\right)^{-1}(E-\hbar \omega)^{-1}(E+\hbar \omega)^{-1} \\
& \times\left[\left(E-\hbar \omega^{\prime}\right)^{-1}+(E-\hbar \omega)^{-1}+(E+\hbar \omega)^{-1}\right] \\
& \left.+\left(E-\hbar \omega^{\prime}\right)^{-1}(E+\hbar \omega)^{-2}\left[\left(E-\hbar \omega^{\prime}\right)^{-1}+2(E+\hbar \omega)^{-1}\right]\right\} \\
& +(-2)\left(\mu_{i_{5}}^{0 u} d_{i_{3}} \mu_{i_{2}}^{u 0} \mu_{i_{4}}^{0 u} \mu_{i_{1}}^{u 0}\right) \\
& \times\left\{\left(E-\hbar \omega-\hbar \omega^{\prime}\right)^{-1}(E-\hbar \omega)^{-2}\left[\left(E-\hbar \omega-\hbar \omega^{\prime}\right)^{-1}+2(E-\hbar \omega)^{-1}\right]\right. \\
& +\left(E-\hbar \omega-\hbar \omega^{\prime}\right)^{-1}(E+\hbar \omega)^{-1}(E-\hbar \omega)^{-1} \\
& \times\left[\left(E-\hbar \omega-\hbar \omega^{\prime}\right)^{-1}+(E+\hbar \omega)^{-1}+(E-\hbar \omega)^{-1}\right] \\
& +\left(E+\hbar \omega+\hbar \omega^{\prime}\right)^{-1}(E+\hbar \omega)^{-1}(E-\hbar \omega)^{-1} \\
& \times\left[\left(E+\hbar \omega+\hbar \omega^{\prime}\right)^{-1}+(E+\hbar \omega)^{-1}+(E-\hbar \omega)^{-1}\right] \\
& \left.+\left(E+\hbar \omega+\hbar \omega^{\prime}\right)^{-1}(E+\hbar \omega)^{-2}\left[\left(E+\hbar \omega+\hbar \omega^{\prime}\right)^{-1}+2(E+\hbar \omega)^{-1}\right]\right\} .
\end{aligned}
$$

The sum of equations (3.8) and (3.12) completely determines the nonlinear response for this particular process. The degeneracy in frequency of two of the photons involved in the process, $\omega_{1}=\omega_{2}$, causes the nonlinear polarizability tensor to present an index symmetry, $O_{i_{1} i_{2} i_{3} i_{4} i_{5}}^{(4)}=O_{\left(i_{1} i_{2}\right) i_{3} i_{4} i_{5}}^{(4)}$. This allows one to express the tensor as a sum of 11 terms. There is no other symmetry present, so the expression for the sum is highly complicated. However, it is greatly simplified when $\omega^{\prime}=\omega$, in which case the five-wave process represents a higher-order correction to SHG. Then, the tensor reduces to a sum of six terms:

$$
\begin{aligned}
O_{i_{1} i_{2} i_{3} i_{4} i_{5}}^{(4)}\left(\omega^{\prime \prime} ;-\right. & \omega, \omega, \omega, \omega) \\
\equiv & O_{\left(i_{1} i_{2} i_{3}\right) i_{4} i_{5}}^{(4)}\left(\omega^{\prime \prime} ;-\omega, \omega, \omega, \omega\right) \\
= & 12 \frac{1}{\left(E^{2}-\hbar^{2} \omega^{2}\right)\left(E^{2}-4 \hbar^{2} \omega^{2}\right)} \\
& \times\left[\mu_{i_{1}}^{0 u} d_{i_{3}} d_{i_{4}} d_{i_{5}} \mu_{i_{2}}^{u 0}+\mu_{i_{2}}^{0 u} d_{i_{1}} d_{i_{4}} d_{i_{5}} \mu_{i_{3}}^{u 0}+\mu_{i_{1}}^{0 u} d_{i_{2}} d_{i_{4}} d_{i_{5}} \mu_{i_{3}}^{u 0}+\mu_{i_{4}}^{0 u} d_{i_{1}} d_{i_{2}} d_{i_{3}} \mu_{i_{5}}^{u 0}\right] \\
& +12 \frac{\left(E^{2}+\hbar^{2} \omega^{2}\right)}{\left(E^{2}-\hbar^{2} \omega^{2}\right)\left(E^{2}-4 \hbar^{2} \omega^{2}\right)\left(E^{2}-9 \hbar^{2} \omega^{2}\right)} \\
& \times\left[\mu_{i_{1}}^{0 u} d_{i_{2}} d_{i_{3}} d_{i_{5}} \mu_{i_{4}}^{u 0}+\mu_{i_{2}}^{0 u} d_{i_{1}} d_{i_{3}} d_{i_{5}} \mu_{i_{4}}^{u 0}+\mu_{i_{3}}^{0 u} d_{i_{1}} d_{i_{2}} d_{i_{5}} \mu_{i_{4}}^{u 0}\right] \\
& +12 \frac{\left(E^{2}+11 \hbar^{2} \omega^{2}\right)}{\left(E^{2}-\hbar^{2} \omega^{2}\right)\left(E^{2}-4 \hbar^{2} \omega^{2}\right)\left(E^{2}-9 \hbar^{2} \omega^{2}\right)} \\
& \times\left[\mu_{i_{1}}^{0 u} d_{i_{2}} d_{i_{3}} d_{i_{4}} \mu_{i_{5}}^{u 0}+\mu_{i_{2}}^{0 u} d_{i_{1}} d_{i_{3}} d_{i_{4}} \mu_{i_{5}}^{u 0}+\mu_{i_{3}}^{0 u} d_{i_{1}} d_{i_{2}} d_{i_{4}} \mu_{i_{5}}^{u 0}\right] \\
& +72 \frac{E^{2}\left(-E^{4}+E^{2} \hbar^{2} \omega^{2}+4 \hbar^{4} \omega^{4}\right)}{\left(E^{2}-\hbar^{2} \omega^{2}\right)^{3}\left(E^{2}-4 \hbar^{2} \omega^{2}\right)^{2}} \\
& \times\left[d_{i_{1}} \mu_{i_{2}}^{u 0} \mu_{i_{3}}^{u 0} \mu_{i_{4}}^{u 0} \mu_{i_{5}}^{u 0}+d_{i_{2}} \mu_{i_{1}}^{u 0} \mu_{i_{3}}^{u 0} \mu_{i_{4}}^{u 0} \mu_{i_{5}}^{u 0}+d_{i_{3}} \mu_{i_{1}}^{u 0} \mu_{i_{2}}^{u 0} \mu_{i_{4}}^{u 0} \mu_{i_{5}}^{u 0}\right]
\end{aligned}
$$


Table 4. Dominant terms in resonance five-wave mixing.

\begin{tabular}{|c|c|c|}
\hline Resonance at & $\Gamma^{-l}$ & \\
\hline \multirow[t]{2}{*}{$E \sim \hbar \omega$} & $l=3$ & $\begin{array}{l}d_{i_{1}} \mu_{i_{2}}^{u 0} \mu_{i_{3}}^{u 0} \mu_{i_{4}}^{u 0} \mu_{i_{5}}^{u 0} ; d_{i_{2}} \mu_{i_{1}}^{u 0} \mu_{i_{3}}^{u 0} \mu_{i_{4}}^{u 0} \mu_{i_{5}}^{u 0} ; d_{i_{3}} \mu_{i_{1}}^{u 0} \mu_{i_{2}}^{u 0} \mu_{i_{4}}^{u 0} \mu_{i_{5}}^{u 0} ; \\
d_{i_{4}} \mu_{i_{1}}^{u 0} \mu_{i_{2}}^{u 0} \mu_{i_{3}}^{u 0} \mu_{i_{5}}^{u 0}\end{array}$ \\
\hline & $l=1$ & $\begin{array}{l}d_{i_{5}} \mu_{i_{1}}^{u 0} \mu_{i_{2}}^{u 0} \mu_{i_{3}}^{u 0} \mu_{i_{4}}^{u 0} ; \mu_{i_{1}}^{0 u} d_{i_{3}} d_{i_{4}} d_{i_{5}} \mu_{i_{2}}^{u 0} ; \mu_{i_{2}}^{0 u} d_{i_{1}} d_{i_{4}} d_{i_{5}} \mu_{i_{3}}^{u 0} \\
\mu_{i_{1}}^{0 u} d_{i_{2}} d_{i_{4}} d_{i_{5}} \mu_{i_{3}}^{u 0} ; \mu_{i_{4}}^{0 u} d_{i_{1}} d_{i_{2}} d_{i_{3}} \mu_{i_{5}}^{u 0} ; \mu_{i_{1}}^{0 u} d_{i_{2}} d_{i_{3}} d_{i_{4}} \mu_{i_{5}}^{u 0} \\
\mu_{i_{2}}^{0 u} d_{i_{1}} d_{i_{3}} d_{i_{4}} \mu_{i_{5}}^{u 0} ; \mu_{i_{3}}^{0 u} d_{i_{1}} d_{i_{2}} d_{i_{4}} \mu_{i_{5}}^{u 0} ; \mu_{i_{1}}^{0 u} d_{i_{2}} d_{i_{3}} d_{i_{5}} \mu_{i_{4}}^{u 0} \\
\mu_{i_{2}}^{0 u} d_{i_{1}} d_{i_{3}} d_{i_{5}} \mu_{i_{4}}^{u 0} ; \mu_{i_{3}}^{0 u} d_{i_{1}} d_{i_{2}} d_{i_{5}} \mu_{i_{4}}^{u 0}\end{array}$ \\
\hline \multirow[t]{2}{*}{$E \sim 2 \hbar \omega$} & $l=2$ & $d_{i_{1}} \mu_{i_{2}}^{u 0} \mu_{i_{3}}^{u 0} \mu_{i_{4}}^{u 0} \mu_{i_{5}}^{u 0} ; d_{i_{2}} \mu_{i_{1}}^{u 0} \mu_{i_{3}}^{u 0} \mu_{i_{4}}^{u 0} \mu_{i_{5}}^{u 0} ; d_{i_{3}} \mu_{i_{1}}^{u 0} \mu_{i_{2}}^{u 0} \mu_{i_{4}}^{u 0} \mu_{i_{5}}^{u 0} ;$ \\
\hline & $l=1$ & $\begin{array}{l}\mu_{i_{1}}^{0 u} d_{i_{3}} d_{i_{4}} d_{i_{5}} \mu_{i_{2}}^{u 0} ; \mu_{i_{2}}^{0 u} d_{i_{1}} d_{i_{4}} d_{i_{5}} \mu_{i_{3}}^{u 0} ; \mu_{i_{1}}^{0 u} d_{i_{2}} d_{i_{4}} d_{i_{5}} \mu_{i_{3}}^{u 0} \\
\mu_{i_{4}}^{0 u} d_{i_{1}} d_{i_{2}} d_{i_{3}} \mu_{i_{5}}^{u 0} ; \mu_{i_{1}}^{0 u} d_{i_{2}} d_{i_{3}} d_{i_{4}} \mu_{i_{5}}^{u 0} ; \mu_{i_{2}}^{0 u} d_{i_{1}} d_{i_{3}} d_{i_{4}} \mu_{i_{5}}^{u 0} \\
\mu_{i_{3}}^{0 u} d_{i_{1}} d_{i_{2}} d_{i_{4}} \mu_{i_{5}}^{u 0} ; \mu_{i_{1}}^{0 u} d_{i_{2}} d_{i_{3}} d_{i_{5}} \mu_{i_{4}}^{u 0} ; \mu_{i_{2}}^{0 u} d_{i_{1}} d_{i_{3}} d_{i_{5}} \mu_{i_{4}}^{u 0} \\
\mu_{i_{3}}^{0 u} d_{i_{1}} d_{i_{2}} d_{i_{5}} \mu_{i_{4}}^{u 0}\end{array}$ \\
\hline$E \sim 3 \hbar \omega$ & $l=1$ & $\begin{array}{l}\mu_{i_{1}}^{0 u} d_{i_{2}} d_{i_{3}} d_{i_{4}} \mu_{i_{5}}^{u 0} ; \mu_{i_{2}}^{0 u} d_{i_{1}} d_{i_{3}} d_{i_{4}} \mu_{i_{5}}^{u 0} ; \mu_{i_{3}}^{0 u} d_{i_{1}} d_{i_{2}} d_{i_{4}} \mu_{i_{5}}^{u 0} ; \\
\mu_{i_{1}}^{0 u} d_{i_{2}} d_{i_{3}} d_{i_{5}} \mu_{i_{4}}^{u 0} ; \mu_{i_{2}}^{0 u} d_{i_{1}} d_{i_{3}} d_{i_{5}} \mu_{i_{3}}^{u 0} ; \mu_{i_{3}}^{0 u} d_{i_{1}} d_{i_{2}} d_{i_{5}} \mu_{i_{4}}^{u 0} ; \\
d_{i_{4}} \mu_{i_{1}}^{u 0} \mu_{i_{2}}^{u 0} \mu_{i_{3}}^{u 0} \mu_{i_{5}}^{u 0} ; d_{i_{5}} \mu_{i_{1}}^{u 0} \mu_{i_{2}}^{u 0} \mu_{i_{3}}^{u 0} \mu_{i_{4}}^{u 0}\end{array}$ \\
\hline
\end{tabular}

$$
\begin{aligned}
& +(-72) \frac{1}{\left(E^{2}-\hbar^{2} \omega^{2}\right)\left(E^{2}-9 \hbar^{2} \omega^{2}\right)} d_{i_{4}} \mu_{i_{1}}^{u 0} \mu_{i_{2}}^{u 0} \mu_{i_{3}}^{u 0} \mu_{i_{5}}^{u 0} \\
& +24 \frac{\left(-3 E^{4}+16 E^{2} \hbar^{2} \omega^{2}+3 \hbar^{4} \omega^{4}\right)}{\left(E^{2}-\hbar^{2} \omega^{2}\right)^{3}\left(E^{2}-9 \hbar^{2} \omega^{2}\right)} d_{i_{5}} \mu_{i_{1}}^{u 0} \mu_{i_{2}}^{u 0} \mu_{i_{3}}^{u 0} \mu_{i_{4}}^{u 0}
\end{aligned}
$$

From this expression it is easy to establish the importance of the different terms in cases of non-secular resonance $(E \sim \hbar \omega, E \sim 2 \hbar \omega$ or $E \sim 3 \hbar \omega)$. In table 4 a summary of the different cases is given. For the first two cases $(E \sim \hbar \omega, E \sim 2 \hbar \omega)$ the main contribution is given by those terms proportional to $\Gamma^{-3}$ and $\Gamma^{-2}$, respectively, while in the third case $(E \sim 3 \hbar \omega)$ all terms have a directly inverse proportionality to the damping factor.

For those molecular systems, in which the transition dipole moment $\mu^{0 u}$ and the vector $\boldsymbol{d}$ differs substantially in magnitude, some of the terms become more relevant than others. If $\left|\mu^{0 u}\right| \ll|d|$, then the most relevant terms are those in the first three terms in equation (3.13). On the other hand, if $\left|\underline{\mu}^{0 u}\right| \gg|\boldsymbol{d}|$, only the last four terms are relevant.

\subsection{Six-wave mixing}

For a six-wave mixing process the number of terms involved is $6 ! \times 2^{5}=23040$, whilst the number of sequences involved is $2^{5}$. These can be grouped as follows:

$p=0: \quad 0000000$

$p=2:$ Оиииии0, Оииии00, ООииии0, Оиии000, О0иии00, О00иии0, Оии0000, О0ии000, 000uи00, 0000uи0, 0u00000, 00u0000, 000u000, 0000u00, 00000u0

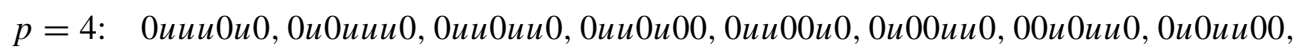
$00 u u 0 u 0,0 u 0 u 000,0 u 00 u 00,0 u 000 u 0,00 u 0 u 00,00 u 00 u 0,000 u 0 u 0$

$p=6: \quad 0 u 0 u 0 u 0$.

The final expression, which is equivalent to that obtained using the transformed interaction Hamiltonian, entails five sequences: Оиииии $0,0 и и и 0 u 0,0 и 0 и и и 0,0 и и 0 u и 0$ and $0 u 0 u 0 u 0$, 
as given by the application of the algorithm (3.1). Thus, the nonlinear polarizability tensor for the six-wave process is written as

$$
\begin{aligned}
\sum_{\pi\{1,2,3,4,5,6\}}( & \left(\mu_{i_{6}}^{0 u} d_{i_{5}} d_{i_{4}} d_{i_{3}} d_{i_{2}} \mu_{i_{1}}^{u 0}\right)\left(E+\sum_{i=1}^{5} \eta_{i} \hbar \omega_{i}\right)^{-1}\left(E+\sum_{i=1}^{4} \eta_{i} \hbar \omega_{i}\right)^{-1} \\
& \times\left(E+\sum_{i=1}^{3} \eta_{i} \hbar \omega_{i}\right)^{-1}\left(E+\sum_{i=1}^{2} \eta_{i} \hbar \omega_{i}\right)^{-1}\left(E+\eta_{1} \hbar \omega_{1}\right)^{-1} \\
& +\left(\mu_{i_{6}}^{0 u} d_{i_{5}} d_{i_{4}} \mu_{i_{3}}^{u 0} \mu_{i_{2}}^{0 u} \mu_{i_{1}}^{u 0}\right)\left(E+\sum_{i=1}^{5} \eta_{i} \hbar \omega_{i}\right)^{-1}\left(E+\sum_{i=1}^{4} \eta_{i} \hbar \omega_{i}\right)^{-1} \\
& \times\left(E+\sum_{i=1}^{3} \eta_{i} \hbar \omega_{i}\right)^{-1}\left(\sum_{i=1}^{2} \eta_{i} \hbar \omega_{i}\right)^{-1}\left(E+\eta_{1} \hbar \omega_{1}\right)^{-1} \\
& +\left(\mu_{i_{6}}^{0 u} \mu_{i_{5}}^{u 0} \mu_{i_{4}}^{0 u} d_{i_{3}} d_{i_{2}} \mu_{i_{1}}^{u 0}\right)\left(E+\sum_{i=1}^{5} \eta_{i} \hbar \omega_{i}\right)^{-1}\left(\sum_{i=1}^{4} \eta_{i} \hbar \omega_{i}\right)^{-1} \\
& \times\left(E+\sum_{i=1}^{3} \eta_{i} \hbar \omega_{i}\right)^{-1}\left(E+\sum_{i=1}^{2} \eta_{i} \hbar \omega_{i}\right)^{-1}\left(E+\eta_{1} \hbar \omega_{1}\right)^{-1} \\
& +\left(\mu_{i_{6}}^{0 u} d_{i_{5}} \mu_{i_{4}}^{u 0} \mu_{i_{3}}^{0 u} d_{i_{2}} \mu_{i_{1}}^{u 0}\right)\left(E+\sum_{i=1}^{5} \eta_{i} \hbar \omega_{i}\right)^{-1}\left(E+\sum_{i=1}^{4} \eta_{i} \hbar \omega_{i}\right)^{-1} \\
& \times\left(\sum_{i=1}^{3} \eta_{i} \hbar \omega_{i}\right)^{-1}\left(E+\sum_{i=1}^{2} \eta_{i} \hbar \omega_{i}\right)^{-1}\left(E+\eta_{1} \hbar \omega_{1}\right)^{-1} \\
& +\left(\mu_{i_{6}}^{0 u} \mu_{i_{5}}^{u 0} \mu_{i_{4}}^{0 u} \mu_{i_{3}}^{u 0} \mu_{i_{2}}^{0 u} \mu_{i_{1}}^{u 0}\right)\left(E+\sum_{i=1}^{5} \eta_{i} \hbar \omega_{i}\right)^{-1}\left(\sum_{i=1}^{4} \eta_{i} \hbar \omega_{i}\right)^{-1} \\
& \left.\times\left(E+\sum_{i=1}^{3} \eta_{i} \hbar \omega_{i}\right)^{-1}\left(\sum_{i=1}^{2} \eta_{i} \hbar \omega_{i}\right)^{-1}\left(E+\eta_{1} \hbar \omega_{1}\right)^{-1}\right)_{\pi}
\end{aligned}
$$

Since this process involves an even number of photons, even for $\boldsymbol{d}=0$ the tensor remains non-zero and is identical to the last term in equation (3.14). On the other hand, if $|d| \gg\left|\underline{\mu^{u 0}}\right|$, then the result can be represented by the first term alone. Again, it is important to notice that for certain six-wave mixing processes secular resonance might be presented when one or more of the following identities is satisfied:

$$
\left(\sum_{i=1}^{2} \eta_{i} \hbar \omega_{i}\right)_{\pi} \cong 0 \quad\left(\sum_{i=1}^{3} \eta_{i} \hbar \omega_{i}\right)_{\pi} \cong 0 \quad \text { and } / \text { or } \quad\left(\sum_{i=1}^{4} \eta_{i} \hbar \omega_{i}\right)_{\pi} \cong 0 .
$$

In any of these cases there will be singularities in some of the last four terms of equation (3.14). For example, when studying SHG in a six-wave mixing process, there are singularities of the second kind. We will study this case in the following subsection.

3.3.1. Particular case. As observed above, with a larger number of photons involved in the process, explicit expression of the nonlinear polarizability tensor produces a rapidly increasing number of terms, which makes it difficult to handle. For cases where the beams involved are characterized by some kind of symmetry this number nevertheless reduces drastically. This is well illustrated by the case of the six-wave mixing process, which effects second harmonic generation in centrosymmetric media $[8,10,11]$. The nonlinearity which mediates 
Table 5. Photon characteristics for a particular six-wave mixing process (the angle between $\hat{k}$ and $\hat{\boldsymbol{k}}^{\prime}$ is arbitrary, and the labels $\lambda, \lambda^{\prime}, \lambda^{\prime \prime}$ and $\lambda^{\prime \prime \prime}$ refer to the polarizations of the beams). The frequencies of the beams involved satisfy the energy conservation condition $\omega^{\prime \prime}=2 \omega$.

\begin{tabular}{llll}
\hline & Frequency & & Direction of propagation \\
\hline Absorbed photons & & & \\
$(\boldsymbol{k}, \lambda)$ & $\omega_{1}=\omega$ & $\eta_{1}=1$ & $\hat{\boldsymbol{k}}$ \\
$(\boldsymbol{k}, \lambda)$ & $\omega_{1}=\omega$ & $\eta_{1}=1$ & $\hat{\boldsymbol{k}}$ \\
$\left(-\boldsymbol{k}, \lambda^{\prime}\right)$ & $\omega_{3}=\omega$ & $\eta_{3}=1$ & $-\hat{\boldsymbol{k}}$ \\
$\left(-\boldsymbol{k}, \lambda^{\prime}\right)$ & $\omega_{4}=\omega$ & $\eta_{4}=1$ & $-\hat{\boldsymbol{k}}$ \\
Emitted photons & & & \\
$\left(\boldsymbol{k}^{\prime \prime}, \lambda^{\prime \prime}\right)$ & $\omega_{5}=\omega^{\prime \prime}$ & $\eta_{5}=-1$ & $\hat{\boldsymbol{k}}^{\prime}$ \\
$\left(-\boldsymbol{k}^{\prime \prime}, \lambda^{\prime \prime \prime}\right)$ & $\omega_{6}=\omega^{\prime \prime}$ & $\eta_{6}=-1$ & $-\hat{\boldsymbol{k}}^{\prime}$ \\
\hline
\end{tabular}

this six-wave interaction can be represented as $O^{(5)}\left(\omega^{\prime \prime} ;-\omega^{\prime \prime}, \omega, \omega, \omega, \omega\right)$, the parameters within parentheses successively represent the harmonic signal photon, the harmonic pump photon, the two photons of one fundamental frequency input and, finally, the two photons of the other (counterpropagating) fundamental input. The beam parameters are fully characterized in table 5 .

The first simplification results from recognition that, since the energy denominators of the tensor are not dependent on the beam direction, the four fundamental frequency photons are interchangeable and so too are the two harmonic photons. This reduces the number of different energy denominators to a set of 15 , the same as those which arise in connection with a much simpler six-wave interaction described previously [10]. Using expression (3.14) for a general six-photon process, the response tensor can be written as a sum of five different terms, each of which involves all the non-equivalent beam permutations. As in the particular case of the five-wave mixing process examined in section 3.2.1, there are some terms involved which have a secular resonance present. This problem has already been solved [17] and we will apply these results for the particular case of a two-level molecular system. Then, $O^{(5)}$ can be written as follows, dividing the contributions from the secular and non-secular parts:

$$
\begin{aligned}
\sum_{\pi\{1,2,3,4,5,6\}}(( & \left.\mu_{i_{6}}^{0 u} d_{i_{5}} d_{i_{4}} d_{i_{3}} d_{i_{2}} \mu_{i_{1}}^{u 0}\right)\left(E+\sum_{i=1}^{5} \eta_{i} \hbar \omega_{i}\right)^{-1}\left(E+\sum_{i=1}^{4} \eta_{i} \hbar \omega_{i}\right)^{-1}\left(E+\sum_{i=1}^{3} \eta_{i} \hbar \omega_{i}\right)^{-1} \\
& \times\left(E+\sum_{i=1}^{2} \eta_{i} \hbar \omega_{i}\right)^{-1}\left(E+\eta_{1} \hbar \omega_{1}\right)^{-1} \\
& +\left(\mu_{i_{6}}^{0 u} \mu_{i_{5}}^{u 0} \mu_{i_{4}}^{0 u} \mu_{i_{3}}^{u 0} \mu_{i_{2}}^{0 u} \mu_{i_{1}}^{u 0}\right)\left(E+\sum_{i=1}^{5} \eta_{i} \hbar \omega_{i}\right)^{-1}\left(\sum_{i=1}^{4} \eta_{i} \hbar \omega_{i}\right)^{-1} \\
& \times\left(E+\sum_{i=1}^{3} \eta_{i} \hbar \omega_{i}\right)^{-1}\left(\sum_{i=1}^{2} \eta_{i} \hbar \omega_{i}\right)^{-1}\left(E+\eta_{1} \hbar \omega_{1}\right)^{-1} \\
& +\left(\mu_{i_{6}}^{0 u} d_{i_{5}} d_{i_{4}} \mu_{i_{3}}^{u 0} \mu_{i_{2}}^{0 u} \mu_{i_{1}}^{u 0}\right)\left(E+\sum_{i=1}^{5} \eta_{i} \hbar \omega_{i}\right)^{-1}\left(E+\sum_{i=1}^{4} \eta_{i} \hbar \omega_{i}\right)^{-1} \\
& \times\left(E+\sum_{i=1}^{3} \eta_{i} \hbar \omega_{i}\right)^{-1}\left(\sum_{i=1}^{2} \eta_{i} \hbar \omega_{i}\right)^{-1}\left(E+\eta_{1} \hbar \omega_{1}\right)^{-1}
\end{aligned}
$$




$$
\begin{aligned}
& +\left(\mu_{i_{6}}^{0 u} \mu_{i_{5}}^{u 0} \mu_{i_{4}}^{0 u} d_{i_{3}} d_{i_{2}} \mu_{i_{1}}^{u 0}\right)\left(E+\sum_{i=1}^{5} \eta_{i} \hbar \omega_{i}\right)^{-1}\left(\sum_{i=1}^{4} \eta_{i} \hbar \omega_{i}\right)^{-1} \\
& \left.\times\left(E+\sum_{i=1}^{3} \eta_{i} \hbar \omega_{i}\right)^{-1}\left(E+\sum_{i=1}^{2} \eta_{i} \hbar \omega_{i}\right)^{-1}\left(E+\eta_{1} \hbar \omega_{1}\right)^{-1}\right)_{\pi} \\
& +\sum_{\substack{\text { non-secular } \\
\pi\{1,2,3,4,5,6\}}}\left(\left(\mu_{i_{6}}^{0 u} d_{i_{5}} \mu_{i_{4}}^{u 0} \mu_{i_{3}}^{0 u} d_{i_{2}} \mu_{i_{1}}^{u 0}\right)\left(E+\sum_{i=1}^{5} \eta_{i} \hbar \omega_{i}\right)^{-1}\left(E+\sum_{i=1}^{4} \eta_{i} \hbar \omega_{i}\right)^{-1}\right. \\
& \left.\times\left(\sum_{i=1}^{3} \eta_{i} \hbar \omega_{i}\right)^{-1}\left(E+\sum_{i=1}^{2} \eta_{i} \hbar \omega_{i}\right)^{-1}\left(E+\eta_{1} \hbar \omega_{1}\right)^{-1}\right)_{\pi} \\
& +\sum_{\substack{\text { secular } \\
p=1, \ldots, 9}} O_{\mathrm{sec} ;(i, j k)(l, m n)}^{(p)}
\end{aligned}
$$

where the sum labelled as 'non-secular, $\pi\{1,2,3,4,5,6\}$ ' is subject to the condition $\left(\sum_{i=1}^{3} \eta_{i} \hbar \omega_{i}\right)_{\pi} \neq 0$. Expression (3.15) shows that, in this case, there is only one term which has secular resonances present. The cases where $\left(\sum_{i=1}^{3} \eta_{i} \hbar \omega_{i}\right)_{\pi}=\hbar \omega^{\prime}-\hbar \omega-\hbar \omega$ or $\left(\sum_{i=1}^{3} \eta_{i} \hbar \omega_{i}\right)_{\pi}=\hbar \omega^{\prime \prime}-\hbar \omega-\hbar \omega$ are contained in the last line of equation (3.15). The explicit expressions for the nine different contributions, given in table 6 , are equivalent to those in [17], but are expressed more concisely here.

Table 6. Secular terms for six-wave SHG

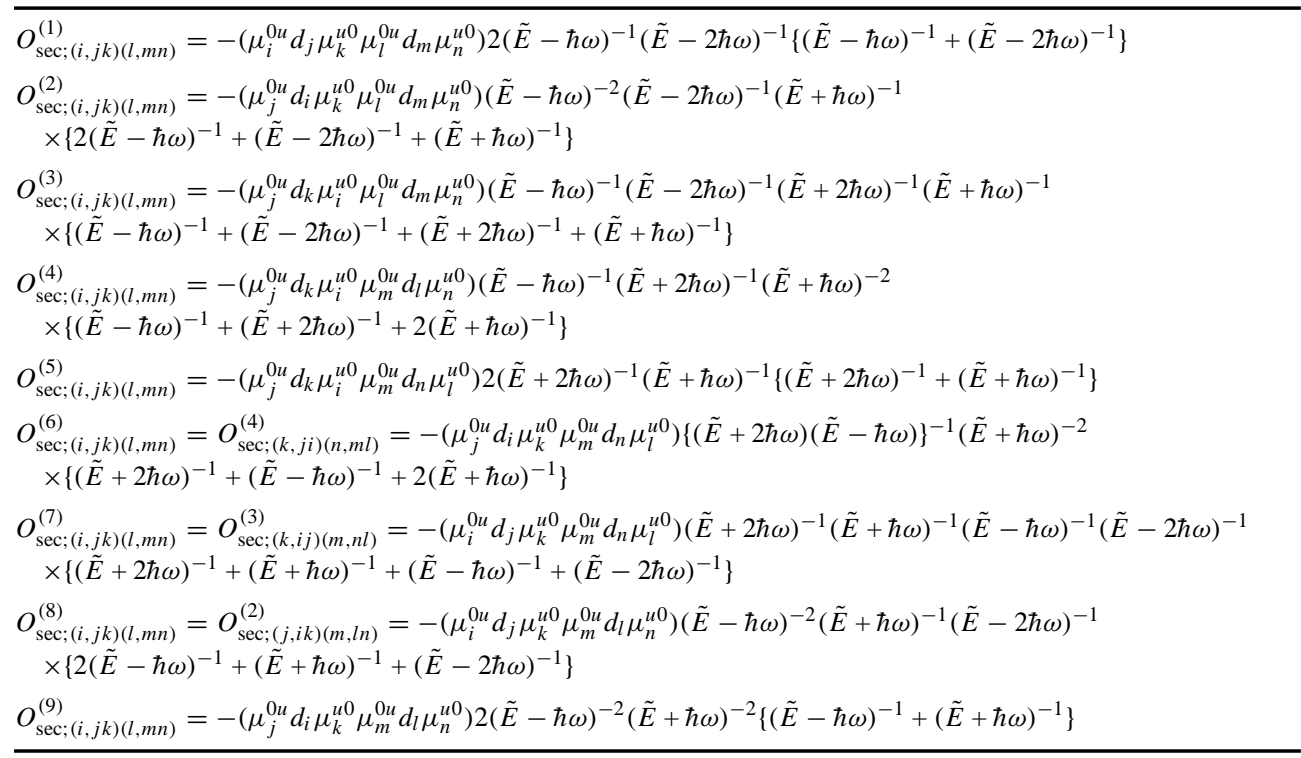

Because this particular case is highly degenerate, the terms of the tensor can be grouped by the type of numerator, of which only six arise: $\mu_{h}^{0 u} \mu_{h}^{0 u} \mu_{f}^{0 u} \mu_{f}^{0 u} \mu_{f}^{0 u} \mu_{f}^{0 u}, \quad \mu_{h}^{0 u} \mu_{h}^{0 u} \mu_{f}^{0 u} \mu_{f}^{0 u} d_{f} d_{f}, \quad d_{h} d_{h} \mu_{f}^{0 u} \mu_{f}^{0 u} \mu_{f}^{0 u} \mu_{f}^{0 u}, \quad \mu_{h}^{0 u} d_{h} \mu_{f}^{0 u} \mu_{f}^{0 u} \mu_{f}^{0 u} d_{f}$, $\mu_{h}^{0 u} d_{h} \mu_{f}^{0 u} d_{f} d_{f} d_{f}, \mu_{h}^{0 u} \mu_{h}^{0 u} d_{f} d_{f} d_{f} d_{f}$ and $d_{h} d_{h} \mu_{f}^{0 u} \mu_{f}^{0 u} d_{f} d_{f}$. Exploiting this feature to gather 
salient terms, the following final expression for the nonlinear polarizability is obtained:

$$
\begin{aligned}
O^{(5)}\left(\omega^{\prime \prime} ;-\omega^{\prime \prime},\right. & \omega, \omega, \omega, \omega)=180 \frac{E}{\left(E^{2}-\hbar^{2} \omega^{2}\right)\left(E^{2}-4 \hbar^{2} \omega^{2}\right)\left(E^{2}-9 \hbar^{2} \omega^{2}\right)} \mu_{h}^{0 u} \mu_{h}^{0 u} \mu_{f}^{0 u} \mu_{f}^{0 u} \mu_{f}^{0 u} \mu_{f}^{0 u} \\
& +(-72) \frac{E\left\{124 \hbar^{8} \omega^{8}+333 \hbar^{6} \omega^{6} E^{2}-156 \hbar^{4} \omega^{4} E^{4}-19 \hbar^{2} \omega^{2} E^{6}+6 E^{8}\right\}}{\left(E^{2}-\hbar^{2} \omega^{2}\right)^{3}\left(E^{2}-4 \hbar^{2} \omega^{2}\right)^{3}\left(E^{2}-9 \hbar^{2} \omega^{2}\right)} \\
& \times \mu_{h}^{0 u} \mu_{h}^{0 u} \mu_{f}^{0 u} \mu_{f}^{0 u} d_{f} d_{f} \\
& +(-24) \frac{E\left(3 E^{2}-11 \hbar^{2} \omega^{2}\right)}{\left(E^{2}-\hbar^{2} \omega^{2}\right)^{3}\left(E^{2}-9 \hbar^{2} \omega^{2}\right)} d_{h} d_{h} \mu_{f}^{0 u} \mu_{f}^{0 u} \mu_{f}^{0 u} \mu_{f}^{0 u} \\
& +96 \frac{\left\{-6 E^{8}+106 E^{6} \omega^{2}-555 E^{4} \omega^{4}+339 E^{2} \omega^{6}+1196 \omega^{8}\right\}}{\left(E^{2}-\hbar^{2} \omega^{2}\right)^{3}\left(E^{2}-4 \hbar^{2} \omega^{2}\right)^{2}\left(E^{2}-9 \hbar^{2} \omega^{2}\right)\left(E^{2}-16 \hbar^{2} \omega^{2}\right)} \mu_{h}^{0 u} d_{h} \mu_{f}^{0 u} \mu_{f}^{0 u} \mu_{f}^{0 u} d_{f} \\
& +(-24) \frac{\left(-2 E^{4}-25 E^{2} \hbar^{2} \omega^{2}+72 \hbar^{4} \omega^{4}\right)}{E\left(E^{2}-\hbar^{2} \omega^{2}\right)\left(E^{2}-4 \hbar^{2} \omega^{2}\right)\left(E^{2}-9 \hbar^{2} \omega^{2}\right)\left(E^{2}-16 \hbar^{2} \omega^{2}\right)} \mu_{h}^{0 u} d_{h} \mu_{f}^{0 u} d_{f} d_{f} d_{f} \\
& +6 \frac{1}{(E)\left(E^{2}-\hbar^{2} \omega^{2}\right)\left(E^{2}-4 \hbar^{2} \omega^{2}\right)} \mu_{h}^{0 U} \mu_{h}^{0 U} d_{f} d_{f} d_{f} d_{f} \\
& +18 \frac{\left(2 E^{2}-3 \hbar^{2} \omega^{2}\right)}{E\left(E^{2}-\hbar^{2} \omega^{2}\right)\left(E^{2}-4 \hbar^{2} \omega^{2}\right)\left(E^{2}-9 \hbar^{2} \omega^{2}\right)} d_{h} d_{h} \mu_{f}^{0 u} \mu_{f}^{0 u} d_{f} d_{f} .
\end{aligned}
$$

From this expression it is noticed that $O^{(5)}$ is proportional to $\left(E^{2}-\hbar^{2} \omega^{2}\right)^{-1}$ and, therefore, if the molecular system is near to single-photon resonance, $E \sim \hbar \omega$, all the terms are enhanced.

\begin{tabular}{|c|c|c|}
\hline Resonance at & $\Gamma^{-l}$ & \\
\hline \multirow[t]{2}{*}{$E \sim \hbar \omega$} & $l=3$ & $\mu_{h}^{0 u} \mu_{h}^{0 u} \mu_{f}^{0 u} \mu_{f}^{0 u} d_{f} d_{f} ; d_{h} d_{h} \mu_{f}^{0 u} \mu_{f}^{0 u} \mu_{f}^{0 u} \mu_{f}^{0 u} ; \mu_{h}^{0 u} d_{h} \mu_{f}^{0 u} \mu_{f}^{0 u} \mu_{f}^{0 u} d_{f}$ \\
\hline & $l=1$ & $\begin{array}{l}\mu_{h}^{0 u} \mu_{h}^{0 u} \mu_{f}^{0 u} \mu_{f}^{0 u} \mu_{f}^{0 u} \mu_{f}^{0 u} ; \mu_{h}^{0 u} d_{h} \mu_{f}^{0 u} d_{f} d_{f} d_{f} ; \mu_{h}^{0 u} \mu_{h}^{0 u} d_{f} d_{f} d_{f} d_{f} \\
d_{h} d_{h} \mu_{f}^{0 u} \mu_{f}^{0 u} d_{f} d_{f}\end{array}$ \\
\hline \multirow[t]{3}{*}{$E \sim 2 \hbar \omega$} & $l=3$ & $\mu_{h}^{0 u} \mu_{h}^{0 u} \mu_{f}^{0 u} \mu_{f}^{0 u} d_{f} d_{f}$ \\
\hline & $l=2$ & $\mu_{h}^{0 u} d_{h} \mu_{f}^{0 u} \mu_{f}^{0 u} \mu_{f}^{0 u} d_{f}$ \\
\hline & $l=1$ & $\begin{array}{l}\mu_{h}^{0 u} \mu_{h}^{0 u} \mu_{f}^{0 u} \mu_{f}^{0 u} \mu_{f}^{0 u} \mu_{f}^{0 u} ; \mu_{h}^{0 u} d_{h} \mu_{f}^{0 u} d_{f} d_{f} d_{f} ; \mu_{h}^{0 u} \mu_{h}^{0 u} d_{f} d_{f} d_{f} d_{f} \\
d_{h} d_{h} \mu_{f}^{0 u} \mu_{f}^{0 u} d_{f} d_{f}\end{array}$ \\
\hline$E \sim 3 \hbar \omega$ & $l=1$ & $\begin{array}{l}\mu_{h}^{0 u} \mu_{h}^{0 u} \mu_{f}^{0 u} \mu_{f}^{0 u} \mu_{f}^{0 u} \mu_{f}^{0 u} ; \mu_{h}^{0 u} \mu_{h}^{0 u} \mu_{f}^{0 u} \mu_{f}^{0 u} d_{f} d_{f} ; d_{h} d_{h} \mu_{f}^{0 u} \mu_{f}^{0 u} \mu_{f}^{0 u} \mu_{f}^{0 u} ; \\
\mu_{h}^{0 u} d_{h} \mu_{f}^{0 u} \mu_{f}^{0 u} \mu_{f}^{0 u} d_{f} ; \mu_{h}^{0 u} d_{h} \mu_{f}^{0 u} d_{f} d_{f} d_{f} ; d_{h} d_{h} \mu_{f}^{0 u} \mu_{f}^{0 u} d_{f} d_{f}\end{array}$ \\
\hline$E \sim 4 \hbar \omega$ & $l=1$ & $\mu_{h}^{0 u} d_{h} \mu_{f}^{0 u} \mu_{f}^{0 u} \mu_{f}^{0 u} d_{f} ; \mu_{h}^{0 u} d_{h} \mu_{f}^{0 u} d_{f} d_{f} d_{f}$ \\
\hline
\end{tabular}
However, the main resonance contributions are given by the second, third and fourth terms, which are proportional to $\Gamma^{-3}$. Enhancement also occurs for $E \sim 2 \hbar \omega, E \sim 3 \hbar \omega, E \sim 4 \hbar \omega$. In the first (two-photon) case, the main contribution to the nonlinear polarizability is given by $\mu_{h}^{0 u} \mu_{h}^{0 u} \mu_{f}^{0 u} \mu_{f}^{0 u} d_{f} d_{f}$. In the other two cases (three- and four-photon resonance) all resonant terms are inversely proportional to $\Gamma$. Table 7 summarizes the terms which are important in each case.

Table 7. Dominant terms in resonance six-wave mixing. 


\section{Discussion}

It has been shown in the previous section that by use of the transformed interaction Hamiltonian, simpler expressions can be found for high orders of the polarizability tensor. These expressions show the dependence of the optical response on the difference between the permanent dipole moment of the excited molecular state $u$ and the ground molecular state 0 , characterizing the associated intramolecular transfer of charge. In general, higher-order processes still present considerable complexity, which is nonetheless greatly reduced for those cases with a high degree of degeneracy amongst the optical modes involved. It has also been shown that the simplest way to obtain the expression for the polarizability tensor given by the transformed Hamiltonian is by the application of the algorithm given in (3.1). This can be achieved for any parametric $n$-photon process as proven in [23].

The broad features of the dependence of the nonlinear polarizability tensor on the permanent dipole moments and the transition dipole moments can be addressed by introducing a factor $\phi$ defined as $\phi=|d|\left|\underline{\mu}^{u 0}\right|^{-1}$, which immediately characterizes the contributions of the different terms in the response tensor. Firstly, let us analyse the simple expression, equation (3.2), relating to the four-wave mixing. For $\phi \gg 1$, the first term of equation (3.2) becomes dominant while, if $\phi \ll 1$, the second term dominates. It is important to notice that the difference in magnitude between the two terms is of the order of $\phi^{2}$, and consequently it is enough to have $\phi \sim 3$, for the first term to become one order of magnitude greater than the second, frequency factors permitting. In the case $\phi \equiv 0$, i.e. where no charge transfer is associated with the optical transition $0 \rightarrow u$, then the nonlinear response remains finite, because, as in any even-photon process, the nonlinear polarizability has a term which is independent of permanent dipole moments. Therefore, for the process under consideration, the nature of the molecular medium determines whether either the first or the second term is dominant.

In a similar manner, the expression for the nonlinear polarizability tensor for five-wave mixing can be written and analysed straightforwardly. The general expression, equation (3.4), shows the dependence of the polarizability on the dipole moments. The first term differs from the rest by $\phi^{2}$, and will therefore become dominant for $\phi \gtrsim 3$. Since this is an odd-photon process, it is forbidden for $\phi=0$ (all terms are dependent on $\boldsymbol{d}$ ). This is a process which very much depends on a strong charge transfer character to the $0 \rightarrow u$ transition. In the specific case of the five-wave process which represents a higher-order correction to SHG, subsection 3.2.1, it is seen that not all non-secular enhancements are of the same order of magnitude. For example, in the single-photon resonance at $E \sim \hbar \omega$, terms which are linearly dependent on the displacement vector $\boldsymbol{d}$ are also proportional to $\Gamma^{-3}$. Similar remarks can be made about the six-wave mixing process, where the five terms can be reduced to just one in the special limits of $\phi \gg 1$ or $\phi \ll 1$. This even-photon process will still generate a signal if $\phi=0$.

In general, for each nonlinear polarizability contribution, the higher the order of the inverse proportionality to the linewidth-related factor $\Gamma$, the greater the magnitude of the corresponding term (since other denominator factors will in general relate to larger electronic energy differences). The significance of the various forms of dependence on the damping can, for example, be illustrated by the case of a two-photon resonant five-wave mixing process. Here, the key contributions to the salient molecular tensor, identified in table 4 , reveal that the $l=2$ contributions carry a lower-order dependence on $d$ than the $l=1$ terms. Thus, unless $\phi$ has a value significantly greater than unity, the $l=2$ terms will certainly dominate. If $\phi$ is much less than unity, the ten $l=1$ terms can effectively be disregarded. Such factors will therefore be important determinants of five-wave optical nonlinearity in molecular systems. 
It is clear that the combined effects of resonance conditions and the interplay of transition/charge transfer dipole moments exercises a considerable effect on the relevance of the various contributions to the nonlinear optical response. The simple expressions we have derived for high-order processes can be used to determine the appropriate characteristics of the nonlinear optical material, which would be necessary to expedite a specific process. Applications to other parametric processes, where there is a particular interest in the dependence on the permanent dipole moments and charge transfer characteristics, can be pursued following the same approach. Although we have limited our interest to those systems with an optical response governed by only two electronic states, an extension to multi-level systems is already being developed.

\section{Acknowledgment}

LDR gratefully acknowledges the award of a research studentship funded by the University of East Anglia (UEA).

\section{References}

[1] Mukamel S 1995 Principles of Nonlinear Optical Spectroscopy (Oxford: Oxford University Press)

[2] Durfee C G III, Backus S, Peatross J, Zeek E, Read K, Weihe F, Murnane M M and Kapteyn H C 1996 Ultrafast Phenomena $X$ (Berlin: Springer) pp 79-80

[3] Sakaguchi H and Nagamura T 1996 Ultrafast Phenomena X (Berlin: Springer) pp 209-10

[4] Steffen T and Duppen K 1996 Ultrafast Phenomena X (Berlin: Springer) pp 213-4

[5] Dubrovskii A V, Koroteev N I and Shkurinov A P 1992 Sov. Phys.-JETP 56551

[6] Shkurinov A P, Dubrovskii A V and Koroteev N I 1993 Phys. Rev. Lett. 701085

[7] Romero L C D, Meech S R and Andrews D L 1997 J. Phys. B: At. Mol. Opt. Phys. 305609

[8] Fiorini C, Charra F and Nunzi J-M 1994 J. Opt. Soc. Am. B 112347

[9] Cho M 1998 J. Chem. Phys. 1096227

[10] Lin S, Andrews D L, Hands I D and Meech S R 1998 Chem. Phys. Lett. 285321

[11] Hands I D, Lin S, Meech S R and Andrews D L 1998 J. Chem. Phys. 10910580

[12] Tominaga K and Yoshihara K 1995 Phys. Rev. Lett. 743061

[13] Tominaga K and Yoshihara K 1996 J. Chem. Phys. 1041159

[14] Tominaga K and Yoshihara K 1996 J. Chem. Phys. 1044419

[15] Steffen T and Duppen K 1996 Phys. Rev. Lett. 761224

[16] Steffen T and Duppen K 1997 J. Chem. Phys. 1063854

[17] Allcock P and Andrews D L 1997 J. Phys. B: At. Mol. Opt. Phys. 303731

[18] Andrews D L 1994 Nonlinear Opt. $822-5$

[19] Meath W J and Power E A 1984 J. Phys. B: At. Mol. Phys. 17763

[20] Kmetic M A and Meath W J 1990 Phys. Rev. A 411556

[21] Andrews D L and Meath W J 1993 J. Phys. B: At. Mol. Opt. Phys. 264633

[22] Craig D P and Thirunamachandran T 1998 Molecular Quantum Electrodynamics: an Introduction to RadiationMolecules Interactions (New York: Dover) p 68

[23] Andrews D L, Romero L C D and Meath W J 1999 J. Phys. B: At. Mol. Opt. Phys. 321

[24] Naguleswaran S and Stedman G E 1996 J. Phys. B: At. Mol. Opt. Phys. 294027

[25] Andrews D L 1993 Modern nonlinear optics Advances in Chemical Physics vol LXXXV, ed M Evans and S Kielich (New York: Wiley) pp 580-1

[26] Bishop D M 1994 J. Chem. Phys. 1006535

[27] Brueckner K A 1955 Phys. Rev. 10036

[28] Dick B and Hohlneicher G 1982 J. Chem. Phys. 765755

[29] Kondo A E, Meath W J, Nilar S H and Thakkar A J 1994 Chem. Phys. 186375

[30] Meath W J and Power E A 1984 Mol. Phys. 51585

[31] Jagatap B N and Meath W J 1996 Chem. Phys. Lett. 258293

[32] Andrews D L, Naguleswaran S and Stedman G E 1998 Phys. Rev. A 574925 